\title{
Tert-butylhydroquinone-decorated graphene nanosheets and their enhanced capacitive behaviors
}

\author{
WANG HuanWen, WU HongYing, CHANG YanQin, CHEN YanLi \& HU ZhongAi* \\ Key Laboratory of Eco-Environment-Related Polymer Materials of Ministry of Education, Key Laboratory of Polymer Materials of Gansu \\ Province, College of Chemistry and Chemical Engineering, Northwest Normal University, Lanzhou 730070, China
}

Received October 16, 2010; accepted December 8, 2010

\begin{abstract}
In the present work, tert-butylhydroquinone (TBHQ) was used to decorate graphene nanosheets to obtain a novel and environmentally friendly electrode material for supercapacitors. The fast redox reactions between hydroquinone and quinone generate pseudocapacitance. Graphene layers which have adsorbed TBHQ interact with each other to construct a three-dimensional network. Through this network, electrolyte ions can easily access the surface of graphene to generate electric double-layer capacitance. Electrochemical measurements have shown that using TBHQ as a redox modifier of graphene can obtain a maximum value of $302 \mathrm{~F} \mathrm{~g}^{-1}$ and provide a $51 \%$ enhancement in specific capacitance. Furthermore, excellent rate capability and cycling ability are achieved using the TBHQ-decorated graphene nanosheet electrode.
\end{abstract}

Graphene, tert-butylhydroquinone, supercapacitor, electrode material

Citation: Wang H W, Wu H Y, Chang Y Q, et al. Tert-butylhydroquinone-decorated graphene nanosheets and their enhanced capacitive behaviors. Chinese Sci Bull, 2011, 56: 2092-2097, doi: 10.1007/s11434-011-4424-0

Supercapacitors have recently attracted wide interest as a possible basis for energy-storage/conversion devices. This is because of their relatively high power density and long life cycle compared with batteries, and their relatively high energy density compared with conventional dielectric capacitors. Such devices could be applied in a large variety of applications, including consumer electronics, memory back-up systems, industrial power, energy management, public transportation, military devices and electric vehicles $[1,2]$. Most research in this area has been focused on the development of electrode materials, such as carbonaceous materials, conducting polymers and transition-metal oxides [3]. Recently, the potential of using graphene-based materials as supercapacitors has received rapidly growing research interest because such materials have excellent electrochemical performance over a wide range of voltage scan rates. Specific capacitances from 10 to $264 \mathrm{~F} \mathrm{~g}^{-1}$ have been obtained for graphene materials in different systems [4-7].

*Corresponding author (email: zhongai@nwnu.edu.cn)
To improve the capacitive behavior of graphene-based materials further, only a few studies have investigated the use of graphene coupled with conducting polymers [8-11]. Also, some researchers are trying to introduce the transition metal oxides/hydroxides with high theoretical specific capacitances into graphene systems [12-16]. In particular, $\mathrm{Ni}(\mathrm{OH})_{2}$ hexagonal nanoplates grown on graphene sheets have shown both high-power and energy capabilities [17]. However, inorganic materials are non-renewable. Their excessive consumption usually causes a series of environmental problems.

There are some organic molecules with reversible electrochemical redox couples that could generate pseudocapacitance under a set of given conditions. For instance, 2-nitro-1-naphthol and anthraquinone have been introduced into carbon black [18] and carbon fabric [19], respectively. In the former case, voltammetric and impedance data obtained in a conventional three-electrode cell were reported, but the modified carbon black was not evaluated as a supercapacitor. In the latter case, the use of anthraquinone as a 
redox modifier for the negative electrode (anode during discharge) of a carbon supercapacitor was shown to provide a $40 \%$ enhancement in average capacitance and $56 \%-86 \%$ increase in energy density. Here, graphene has superior electrical conductivities and high specific areas (theoretically $2630 \mathrm{~m}^{2} \mathrm{~g}^{-1}$ ), and it is a conjugated system. Therefore, graphene adsorbing hydroquinone derivatives at the appropriate surface coverage could be expected to produce an enhanced electrochemical capacitance.

In the present work, we select TBHQ to serve as the electrochemically active molecule to demonstrate the feasibility of this idea. The benzene ring acts as $\pi-\pi$ stack with graphene, and the tert-butyl enhances the hydrophobicity, which leads to the immobilization of the TBHQ on graphene sheets. As a result, quinone/hydroquinone redox couples are introduced into the surfaces of the graphene nanosheets (GNSs) through non-covalent modification, which does not disrupt the $\mathrm{sp}^{2}$ network of the graphene, and superimpose their pseudocapacitance on the electric double-layer capacitance of the graphene.

\section{Experimental}

\subsection{Synthesis of the TBHQ-decorated GNS}

Graphite oxide (GO) was synthesized from graphite powder using a modified Hummers method [20,21]. To obtain the TBHQ-decorated GNSs, $200 \mathrm{mg}$ of GO was dissolved in $100 \mathrm{~mL}$ of water via ultrasonic treatment for $1 \mathrm{~h}$, and 0.02 $\mathrm{mg}$ of TBHQ was dissolved in $10 \mathrm{~mL}$ of ethanol. The above solution was added into the GO suspension using a dropper while being vigorously stirred for $24 \mathrm{~h}$. Then, $4 \mathrm{~g}$ of $\mathrm{NaBH}_{4}$ was slowly added to the mixture while continuously stirring for $24 \mathrm{~h}$ at room temperature. Finally, the resulting product was separated using suction filtration and rinsed with distilled water several times. It was then dried at $60^{\circ} \mathrm{C}$ for $12 \mathrm{~h}$ in vacuum. For comparison, pure graphene was synthesized using the same procedure in the absence of TBHQ.

\subsection{Characterization methods}

The structure and morphology of the samples were characterized using X-ray diffraction (XRD; D/Max-2400, Rigaku, Tokyo, Japan), field emission scanning electron microscopy (FESEM; JEOL, JSM-6701F, Tokyo, Japan) and transmission electron microscopy (TEM; JEOL, JEM-2010). The components of the materials were measured using a Nicolet Nexus 670 FT-IR (Thermo Scientific, Waltham, MA, USA).

\subsection{Preparation of electrodes and electrochemical measurement}

The working electrodes were prepared by mixing the electroactive material, acetylene black, conducting graphite and polytetrafluoroethylene (PTFE) binder in a mass ratio of $75: 10: 10: 5$ to obtain a slurry. The slurry was pressed onto the graphite current collector and dried at $60^{\circ} \mathrm{C}$ for $12 \mathrm{~h}$ in vacuum. Each working electrode contained about $3 \mathrm{mg}$ of the electroactive material and had a surface area of about $1 \mathrm{~cm}^{2}$.

A typical three-electrode experimental cell equipped with a working electrode, a platinum foil counter electrode, and a saturated calomel electrode reference electrode was used to measure the electrochemical properties of the working electrodes. All the electrochemical measurements were carried out in a $1 \mathrm{~mol} \mathrm{~L}^{-1} \mathrm{H}_{2} \mathrm{SO}_{4}$ aqueous electrolyte at room temperature. Cyclic voltammograms (CV) and galvanostatic charge-discharge curve were measured using a CHI 660B electrochemical workstation (CHI Instruments, Austin, TX, USA).

\section{Results and discussion}

\subsection{Material characterization}

The basal planes of graphene oxide sheets are decorated primarily with epoxy and hydroxyl groups, with carboxyl groups located at the edges [14,22]. Specifically, because of the introduction of oxygen-containing functional groups on the carbon nanosheets, the adsorption and intercalation of molecules and ions is possible [23]. Therefore, in our experiment, TBHQ was first adsorbed on graphene oxide sheets in aqueous solution via sonication, which formed a homogeneous suspension. Then, the mixture was reduced using $\mathrm{NaBH}_{4}$ to yield the TBHQ-decorated GNS. A schematic of the formation process of this hybrid material is shown in Figure 1.

The morphology of the TBHQ-decorated GNS was characterized using FESEM and TEM. FESEM and TEM images of the TBHQ-decorated GNSs are shown in Figure 2. From the FESEM image (Figure 2(a)), it can be seen that graphene layers interact with each other to form an open pore network. Through this network the electrolyte ions can easily access the surface of the graphene to form electric double layers. The TEM image (Figure 2(b)) shows that individual graphene sheets are curled and entangled. From Figure 2(c), the two graphene layers in a stack can be seen. This implies that multilayered graphene sheets coexist with individual graphene sheets.

The XRD pattern (Figure 3(a)) shows that the GO has a dominant peak centered at $2 \theta=10.3^{\circ}$. This corresponds to the (001) plane with an inter-planar spacing of $8.6 \AA$. After reduction with $\mathrm{NaBH}_{4}$, this peak completely disappears, but a broad peak (002) centered at around $23.2^{\circ}$ can be seen. This corresponds to an interlayer spacing of $0.38 \mathrm{~nm}$. This indicates that the GO is reduced to graphene in the reaction process.

Fourier-transform infrared spectrometry (FTIR) traces of the GO, GNS and TBHQ-decorated GNS are shown in 


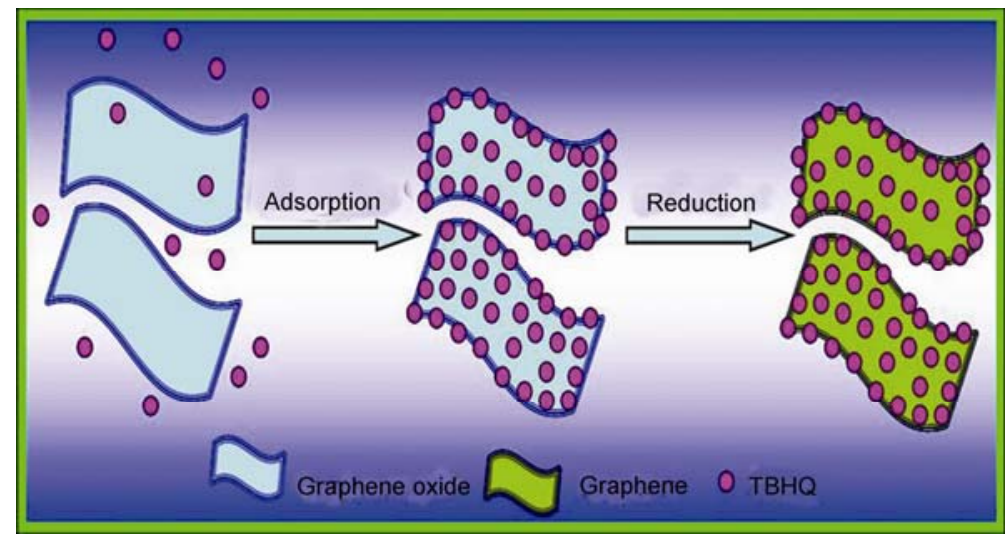

Figure 1 Schematic of the synthesis of the TBHQ-decorated GNS.
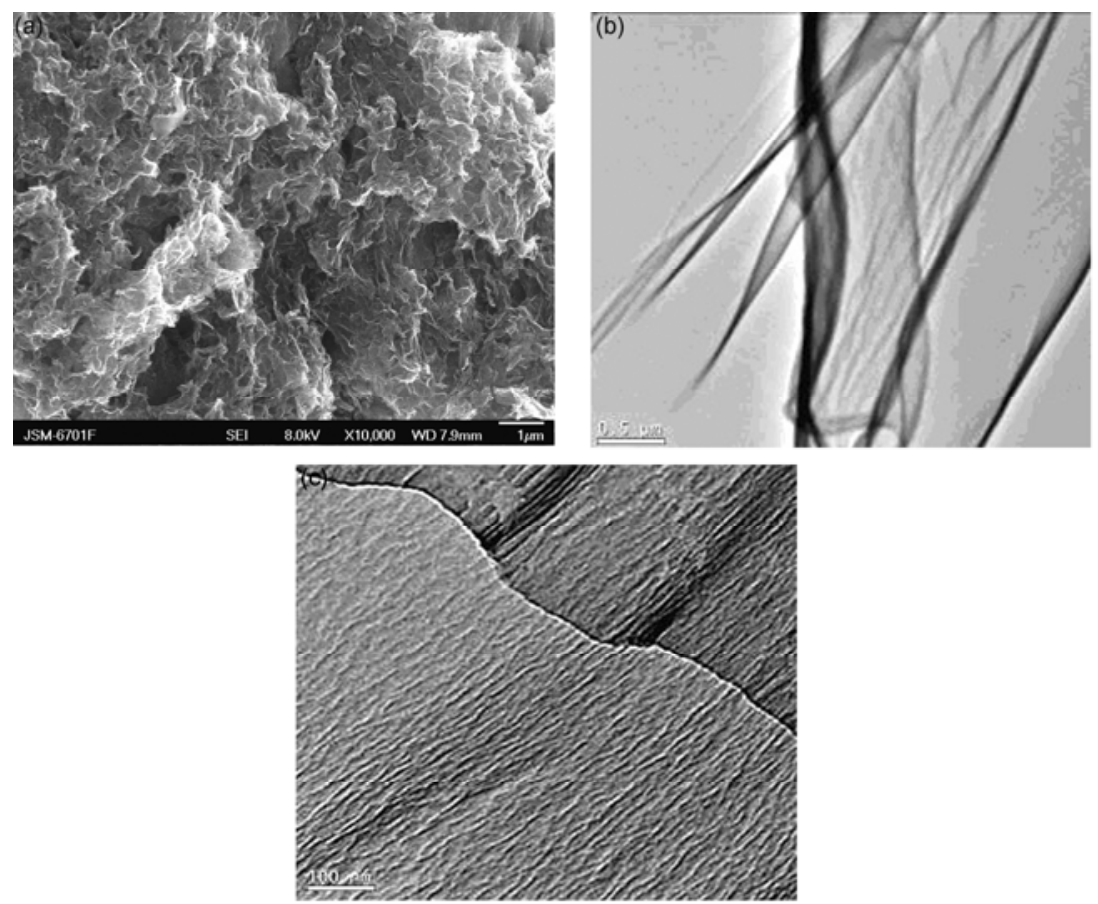

Figure 2 FESEM (a) and TEM ((b),(c)) images of the TBHQ-decorated GNS.

Figure 3(b). In the FTIR spectra of the GO, the peaks at 3400 and $1624 \mathrm{~cm}^{-1}$ are attributed to $\mathrm{O}-\mathrm{H}$ stretching and bending vibrations, respectively. The other oxygencontaining functional groups are represented by the bands at $1725,1065,1245$ and $1375 \mathrm{~cm}^{-1}$, which correspond to $\mathrm{C}=\mathrm{O}$ in $\mathrm{COOH}, \mathrm{C}-\mathrm{O}, \mathrm{C}-\mathrm{OH}$ and $\mathrm{C}-\mathrm{O}-\mathrm{C}$ (epoxy), respectively [24]. Compared with the peaks of GO, all these bands almost vanish in the FTIR spectra of the GNS. This supports the assertion that GO was transformed into GNS during the reaction process. Also, the new peak at $1572 \mathrm{~cm}^{-1}$ in the GNS can be attributed to the skeletal vibration of the GNS. In Figure 3(b), it can be seen that the FTIR spectrum of the TBHQ-decorated GNS is similar to that of GNS, except for the peaks near $2960 \mathrm{~cm}^{-1}$, which are due to $\mathrm{C}-\mathrm{H}$ stretching vibrations from TBHQ. Also, the other function groups in TBHQ are not represented in the IR spectrum of the TBHQ-decorated GNS. This may be because of the low TBHQ content in the composite or low transmittance in presence of GNS.

\subsection{Electrochemical characterization}

$\mathrm{CV}$ and galvanostatic charge-discharge measurements were used to evaluate the electrochemical properties and quantify the specific capacitance of the materials prepared using the above-mentioned process. Figure 4(a) shows the CV measurements for the GNS and TBHQ-decorated GNS electrodes at $2 \mathrm{mV} \mathrm{s}^{-1}$ in the range of $0.0-0.8 \mathrm{~V}$. It can be seen that the CV curve of the GNSs exhibits the typical rectangular shape. This indicates good charge propagation at the 

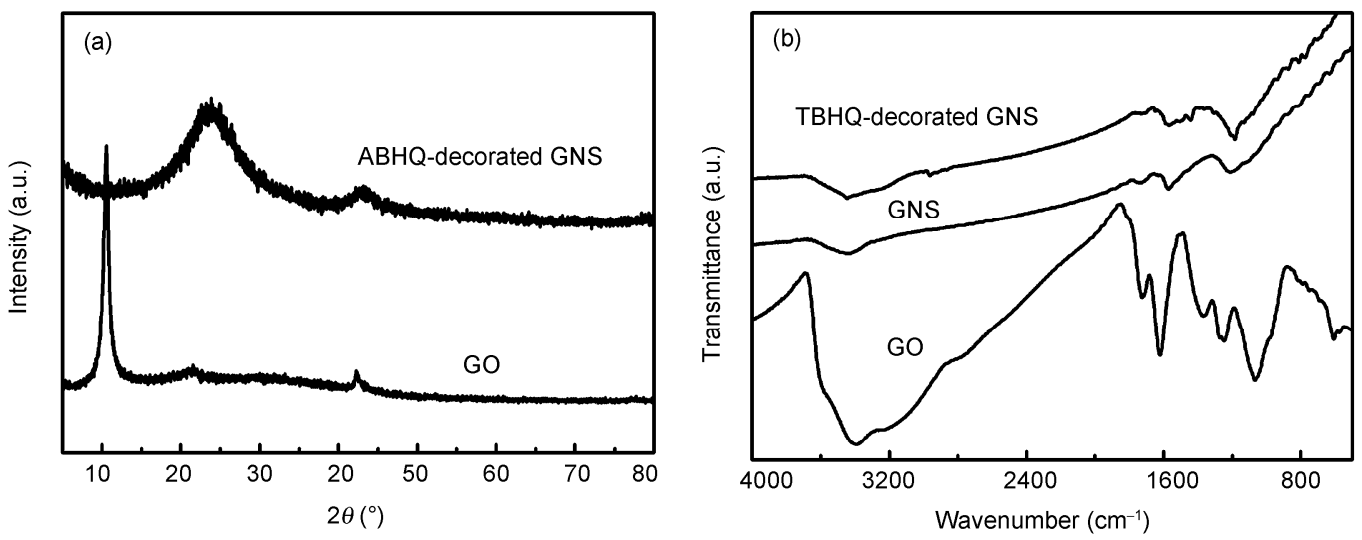

Figure 3 (a) XRD patterns of GO and the TBHQ-decorated GNS. (b) FTIR spectra of GO, GNS and the TBHQ-decorated GNS.

electrode surface following the electric-double-layer charging mechanism. Compared with the GNS, the CV curve of the TBHQ-decorated GNS shows well-defined and reversible redox peaks at 0.415 and $0.366 \mathrm{~V}$. These peaks have symmetric wave-shapes and a small peak separation of 49 $\mathrm{mV}$, which is expected for surface confined redox species. This result also shows that electron transfer between the electroactive sites of TBHQ and graphene matrix is a fast process. The corresponding redox reaction via a coupled proton-electron transfer is shown below:

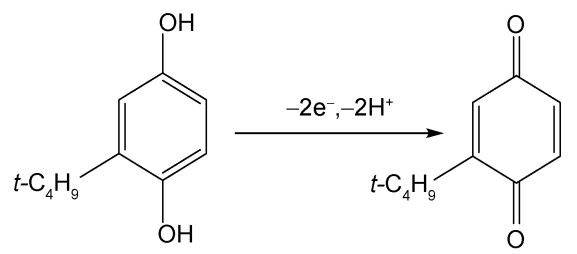

Hydroquinone undergoes oxidation becomes quinone in the charge step, and reduction of quinone reverts it to hydroquinone in the discharge step. The $\pi-\pi$ stacking interaction can make the benzene ring of TBHQ parallel to the surface of the graphene. In this interaction model, the dis- tance between the conductive substrate and the electrochemically active sites is short, and consequently the charge-transfer resistance is low. Hence, fast redox reactions between hydroquinone and quinone could occur at the lower overpotentials. In addition, we also found (Figure 4(a)) that the background current of the TBHQ-decorated GNS is larger than that of the GNS, which indicates that there are positive synergic effects between the two components (TBHQ and graphene).

Figure 4(b) shows discharge curves of the GNS and TBHQ-decorated GNS in the potential range from 0 to $0.8 \mathrm{~V}$ at a specific current of $0.25 \mathrm{~A} \mathrm{~g}^{-1}$. For the GNS electrode, discharge duration is ascribed to the electric double-layer capacitance. For the TBHQ-decorated GNS electrode, the combination of the electric double-layer capacitance and Faradaic capacitance is responsible for the increased discharge duration. These are caused by faradaic charge-transfer accompanied by the double-layer discharging process. These results are in accordance with those inferred from the $C-V$ tests. The specific capacitance was calculated using $C=I \times t /(V \times m)$, where $I$ is the discharge current, $t$ is the discharge time, $V$ is the potential drop during discharge, and $m$ is the mass of active material in a single
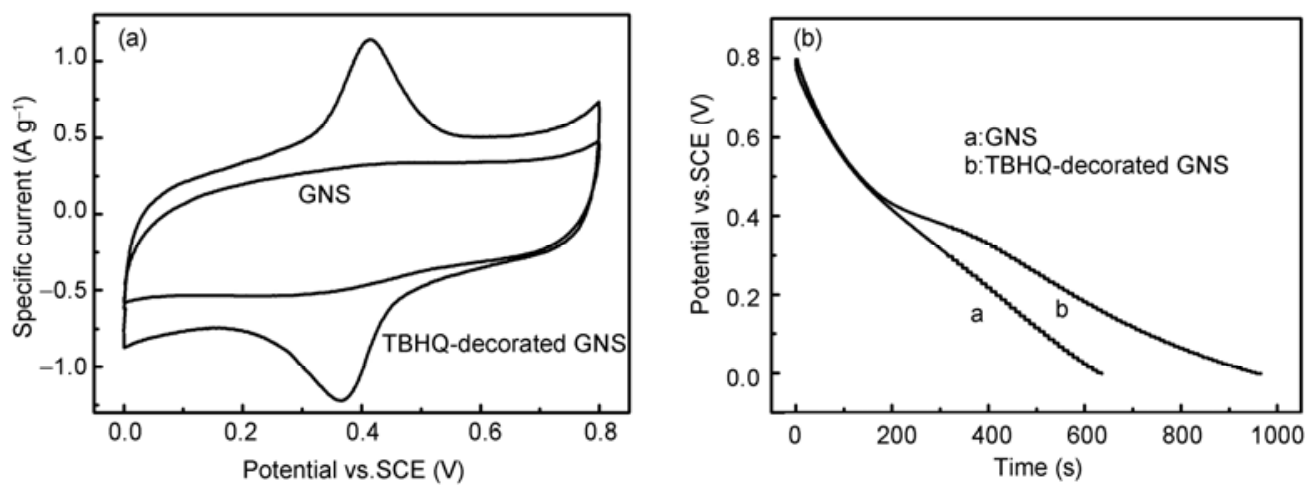

Figure 4 (a) Cyclic voltammograms of the GNS and TBHQ-decorated GNS at $2 \mathrm{mV} \mathrm{s}^{-1}$. (b) Discharge curves of the GNS and TBHQ-decorated GNS at $0.25 \mathrm{~A} \mathrm{~g}^{-1}$. 
electrode. The specific capacitance values were calculated to be 200 and $302 \mathrm{~F} \mathrm{~g}^{-1}$ for the GNS and TBHQ-decorated GNS electrodes, respectively. Assuming that the GNSs in the composite still hold their specific capacitance values, we estimated the TBHQ component to be $8.1 \%$ of the total mass associated with the theoretical specific capacitance (1451.6 $\mathrm{F} \mathrm{g}^{-1}$ ) of TBHQ. In other words, a loading of only $8.1 \%$ of TBHQ on the graphene sheets provides $51 \%$ enhancement in the specific capacitance. Note that this value (302 $\mathrm{F} \mathrm{g}^{-1}$ ) is higher than that of graphene obtained using electrophoretic deposition (164 $\mathrm{F} \mathrm{g}^{-1}$ ) [5]. Our materials also outperform graphene exfoliated via low-temperature vacuum (264 $\mathrm{F} \mathrm{g}^{-1}$ ) [6]. Therefore, enhancing the capacitive behavior of graphene material through non-covalent modification with organic molecules is an effective method.

Figure 5(A) shows the cyclic voltammograms of the TBHQ-decorated GNS electrode at different sweep rates. It can be seen that the cyclic voltammograms retain a similar shape for the entire sweep. This indicates that the material has excellent capacitance behavior, and the electrolyte ions can quickly diffuse into the graphene network. In addition, the TBHQ-decorated GNS also exhibits superior rate capabilities, which can be seen in Figures 5(B) and (C). At a specific current of $8 \mathrm{~A} \mathrm{~g} \mathrm{~g}^{-1}$, the specific capacitance of the TBHQ-decorated GNS is $190 \mathrm{~F} \mathrm{~g}^{-1}, 63 \%$ of that at 0.25 $\mathrm{A} \mathrm{g}^{-1}$. In comparison, the specific capacitance of the GNS decreases to only $50 \mathrm{~F} \mathrm{~g}^{-1}$ at $8 \mathrm{~A} \mathrm{~g}^{-1}, 25 \%$ of that at $0.25 \mathrm{~A} \mathrm{~g}^{-1}$.
To evaluate the stability of the TBHQ-decorated GNS electrode, the values of specific capacitance with respect to the cycle number (up to 800 cycles) at $1 \mathrm{~A} \mathrm{~g}^{-1}$ were measured. This is shown in Figure 5(D). It was found that the TBHQ-decorated GNS electrode exhibits a long cycle life. It retained $94 \%$ specific capacitance after 800 cycle tests. This demonstrates that the charge-discharge process does not induce microstructural changes in the materials. The long-term stability suggests that TBHQ-decorated GNSs could be a promising electrode material for supercapacitors.

\section{Conclusions}

In summary, the non-covalent modification of graphene sheets through organic molecules with reversible redox couples improves their capacitive behavior, rate capability and cycle life. The use of TBHQ as a redox modifier of graphene is shown to obtain a maximum value of $302 \mathrm{~F} \mathrm{~g}^{-1}$ and provides a $51 \%$ enhancement in specific capacitance. These results suggest that the strategy used here is an effective route for designing organic molecule electrode materials for supercapacitors.

The work was supported by the National Natural Science Foundation of China (20963009), the Gansu Science and Technology Committee (0803RJA005) and the postgraduate advisor program of the Provincial
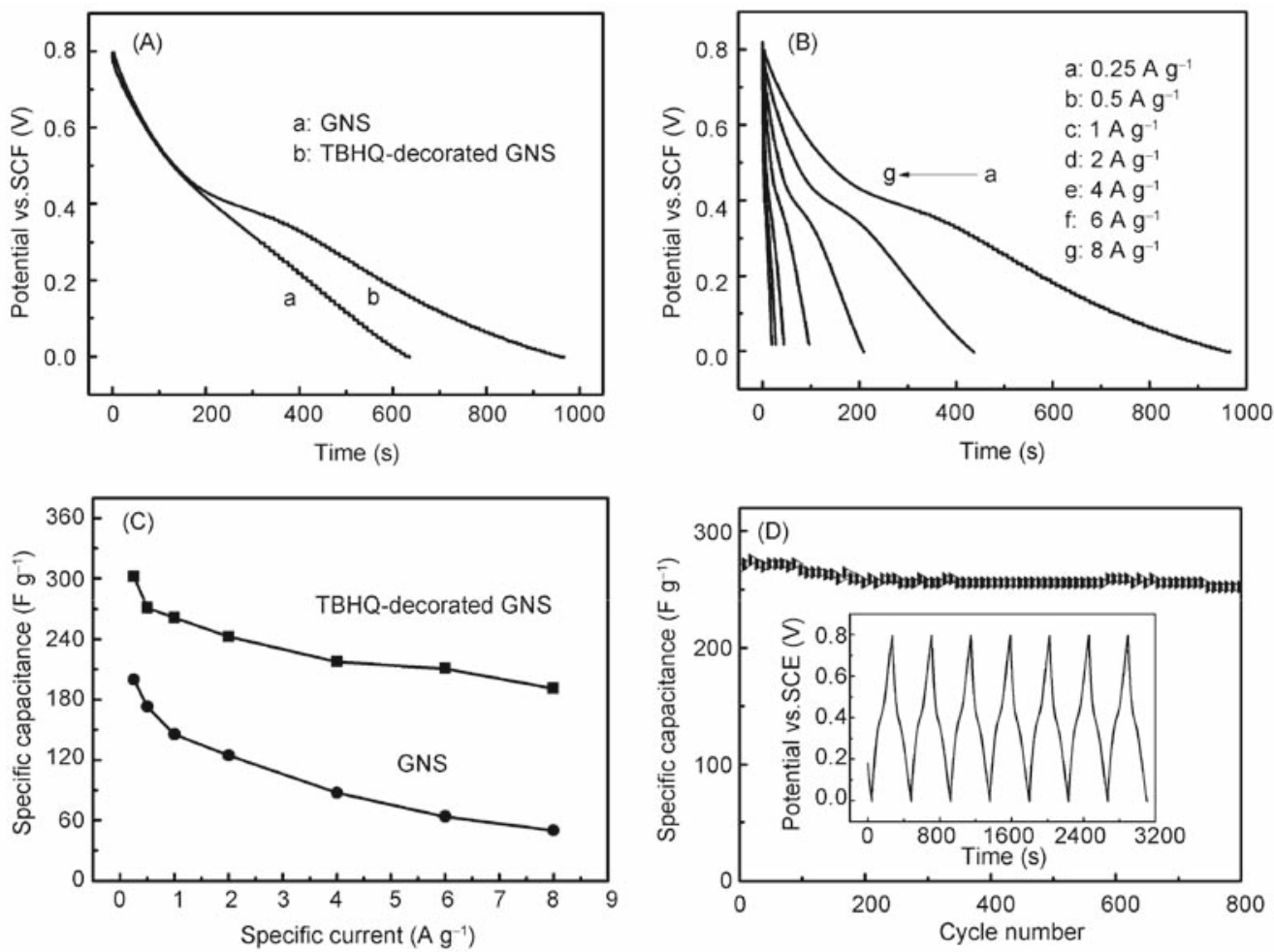

Figure 5 (A) Cyclic voltammograms of the TBHQ-decorated GNSs at different sweep rates. (B) Discharge curves of the TBHQ-decorated GNS at different specific currents. (C) Specific capacitances of the GNSs and TBHQ-decorated GNSs at different specific currents. (D) Cycle life of the TBHQ-decorated GNSs measured at $1 \mathrm{~A} \mathrm{~g}^{-1}$ and its galvanostatic charge-discharge curve (inset). 


\section{Education Department of Gansu.}

1 Conway B E. Transition from supercapacitor to battery behavior in electrochemical energy storage. J Electrochem Soc, 1991, 138: 1539-1548

2 Bi R R, Wu X L, Cao F F, et al. Highly dispersed $\mathrm{RuO}_{2}$ nanoparticles on carbon nanotubes: Facile synthesis and enhanced supercapacitance performance. J Phys Chem C, 2010, 114: 2448-2451

3 Li G R, Feng Z P, Ou Y N, et al. Mesoporous $\mathrm{MnO}_{2} /$ carbon aerogel composites as promising electrode materials for high-performance supercapacitors. Langmuir, 2010, 26: 2209-2213

4 Stoller M D, Park S, Zhu Y, et al. Graphene-based ultracapacitors. Nano Lett, 2008, 8: 3498-3502

5 Chen Y, Zhang X, Yu P, et al. Electrophoretic deposition of graphene nanosheets on nickel foams for electrochemical capacitors. J Power Sources, 2010, 195: 3031-3035

6 Lv W, Tang D M, He Y B, et al. Low-temperature exfoliated graphenes: Vacuum-promoted exfoliation and electrochemical energy storage. ACS Nano, 2009, 3: 3730-3736

7 Zhu Y W, Stoller M D, Cai W W, et al. Exfoliation of graphite oxide in propylene carbonate and thermal reduction of the resulting graphene oxide platelets. ACS Nano, 2010, 4: 1227-1233

8 Zhang K, Zhang L L, Zhao X S, et al. Graphene/polyaniline nanofiber composites as supercapacitor electrodes. Chem Mater, 2010, 22: 1392-1401

9 Yan J, Wei T, Shao B, et al. Preparation of a graphene nanosheet/ polyaniline composite with high specific capacitance. Carbon, 2010, 48: 487-493

10 Murugan A V, Muraliganth T, Manthiram A. Rapid, facile microwave-solvothermal synthesis of graphene nanosheets and their polyaniline nanocomposites for energy storage. Chem Mater, 2009, 21: 5004-5006

11 Wang D W, Li F, Zhao J, et al. Fabrication of graphene/polyaniline composite paper via in situ anodic electropolymerization for highperformance flexible electrode. ACS Nano, 2009, 3: 1745-1752

12 Zhang Y, Li H, Pan L, et al. Capacitive behavior of grapheme-ZnO composite film for supercapacitors. J Electroanal Chem, 2009, 634: 68-71
13 Lu T, Zhang Y, Li H, et al. Electrochemical behaviors of graphene- $\mathrm{ZnO}$ and grapheme- $\mathrm{SnO}_{2}$ composite films for supercapacitors. Electrochim Acta, 2010, 55: 4170-4173

14 Chen $\mathrm{S}$, Zhu J, Wu X, et al. Graphene oxide- $\mathrm{MnO}_{2}$ nanocomposites for supercapacitors. ACS Nano, 2010, 4: 2822-2830

15 Yan J, Fan Z, Wei T, et al. Fast and reversible surface redox reaction of grapheme- $\mathrm{MnO}_{2}$ composites as supercapacitor electrodes. Carbon, 2010, 48: 3825-3833

16 Wang B, Park J, Wang C, et al. $\mathrm{Mn}_{3} \mathrm{O}_{4}$ nanoparticles embedded into graphene nanosheets: Preparation, characterization, and electrochemical properties for supercapacitors. Electrochim Acta, 2010, 55: 6812-6817

17 Wang $\mathrm{H}$, Casalongue $\mathrm{H} \mathrm{S}$, Liang $\mathrm{Y}$, et al. $\mathrm{Ni}(\mathrm{OH})_{2}$ nanoplates grown on graphene as advanced electrochemical pseudocapacitor materials. J Am Chem Soc , 2010, 132: 7472-7477

18 Leitner K W, Gollas B, Winter M, et al. Combination of redox capacity and double layer capacitance in composite electrodes through immobilization of an organic redox couple on carbon black. Electrochim Acta, 2004, 50: 199-204

19 Kalinathan K, DesRoches D P, Liu X, et al. Anthraquinone modified carbon fabric supercapacitors with improved energy and power densities. J Power Sources, 2008, 181: 182-185

20 Hummers W S, Offeman R E. Preparation of graphitic oxide. J Am Chem Soc, 1958, 80: 1339

21 Kovtyukhova N I, Ollivier P J, Martin B R, et al. Layer-by-layer assembly of ultrathin composite films from micron-Sized graphite oxide sheets and polycations. Chem Mater, 1999, 11: 771-778

22 Lee D W, De Los Santos V L, Seo J W, et al. The structure of graphite oxide: Investigation of its surface chemical groups. J Phys Chem B, 2010, 114: 5723-5728

23 Scheuermann G M, Rumi L, Steurer P, et al. Palladium nanoparticles on graphite oxide and its functionalized graphene derivatives as highly active catalysts for the Suzuki-Miyaura coupling reaction. J Am Chem Soc, 2009, 131: 8262-8270

$24 \mathrm{Xu} \mathrm{Y,} \mathrm{Bai} \mathrm{H,} \mathrm{Lu} \mathrm{G,} \mathrm{et} \mathrm{al.} \mathrm{Flexible} \mathrm{graphene} \mathrm{films} \mathrm{via} \mathrm{the} \mathrm{filtration} \mathrm{of}$ water-soluble noncovalent functionalized graphene sheets. J Am Chem Soc, 2008, 130: 5856-5857

Open Access This article is distributed under the terms of the Creative Commons Attribution License which permits any use, distribution, and reproduction in any medium, provided the original author(s) and source are credited. 